\title{
Optical Cladding Waveguides in Dielectric Crystals Produced by Femtosecond Laser Inscription
}

\author{
Feng Chen \\ School of Physics, State Key Laboratory of Crystal Materials and Key Laboratory of Particle Physics and Particle Irradiation (MOE), Shandong \\ University, Jinan 250100, China \\ Author e-mail address: drfchen@sdu.edu.cn
}

\begin{abstract}
In this work, the recent progress of our research on optical cladding waveguides in dielectric crystals produced by femtosecond laser inscription has been overviewed. With different scales at cross sections, the cladding waveguides support guidance from single mode to highly multi-modes, and work for wavelength till mid-infrared regimes. Applications of the fabricated cladding structures as new integrated light sources are introduced.
\end{abstract}

\section{Introduction}

As the basic elements in integrated photonics, optical waveguide structures can confine the light propagation in small volumes, achieving high optical intensities with respect to bulk materials, in which high-performance photonic devices could be constructed in small, compact circuits [1]. Femtosecond (fs) laser inscription has become a powerful and unique technique to fabricate waveguides in a wide range of optical materials [2], since Davis et al. reported their pioneer work in glass [3]. Depending on the diverse parameters of the fs laser pulses and the material properties, the fs-laser inscribed waveguides can be with directly written structures (so-called Type I waveguide with single line writing) [4], stress-induced waveguides (Type II with double line filaments) [5], and depressed cladding waveguides [6]. In the cladding structures, the waveguide are located in the regions surrounded by the fslaser induced tracks with negative refractive-index changes. As of yet, people have fabricated cladding structures in a couple of dielectric crystals and ceramics. One of the advantages of the cladding waveguides is the designable shapes of the configurations and flexible diameters of the cross sections, which makes the cladding structures easily connected with the optical fibers, and support guidance till very long wavelength in Mid-IR regimes. In addition, it has been proved that the cladding waveguide usually offer guidance at two transverse polarizations, which is very important for phase matched frequency doubling.

In this work, the recent progress of our research on optical cladding waveguides in dielectric crystals produced by fs laser inscription, including the fabrication and applications, will be summarized.

\section{Fabrication}

In our work, dielectric crystal cladding waveguides were produced by using an amplified Ti:Sapphire laser system at the Universidad de Salamanca, Spain, or by using a Fiber laser at Heriot-Watt University, UK. Figure 1 shows the schematic plot of the typical writing of cladding waveguides in crystals.

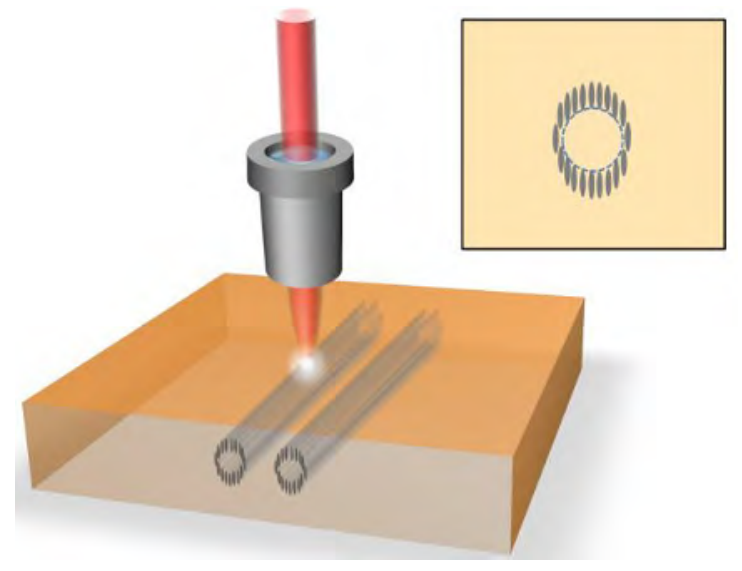

Fig. 1. The schematic of the cladding waveguide fabrication process.

The waveguide core is within the region surrounded by fs-laser inscribed tracks, in which the refractive index is reduced with respect to the bulk. The writing speed of the fs-laser spot is set up 0.7-1 mm/s, which offers fast 
fabrication with acceptable guiding qualities. By using this technique, we have produced cladding waveguides in a couple of dielectric crystals, including laser media $\mathrm{Nd}: \mathrm{YVO}_{4}, \mathrm{Nd}: \mathrm{GdVO}_{4}, \mathrm{Nd}: \mathrm{YAG}, \mathrm{Nd}: \mathrm{GGG}$ and nonlinear crystals $\mathrm{KTiOPO}_{4}, \mathrm{BiB}_{3} \mathrm{O}_{6}, \beta-\mathrm{BaB}_{2} \mathrm{O}_{4}$, etc [7-10].

\section{Waveguide lasers}

Benefiting from the compact geometry, waveguide lasers are usually with reduced lasing thresholds and comparable efficiency with bulk laser systems. We have achieved continuous wave lasing in the fs-laser inscribed cladding waveguides. Most of them have shown high performance laser features. Figure 2 depicts the laser emission spectrum from the fs-laser inscribed $\mathrm{Nd}: \mathrm{YVO}_{4}$ cladding waveguide (120 $\mu \mathrm{m}$ diameter), when the absorbed power is above the lasing threshold.

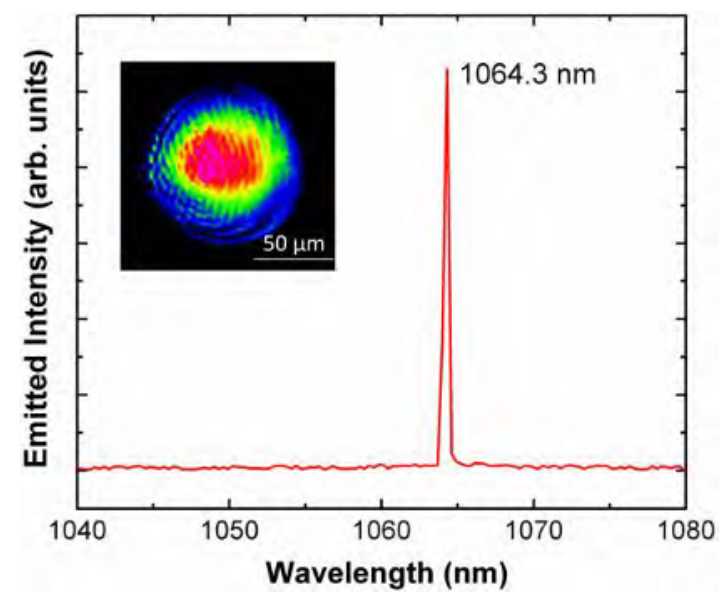

Fig. 2 Laser emission spectrum from the fs-laser inscribed $\mathrm{Nd}: \mathrm{YVO}_{4}$ cladding waveguide (120 $\mu \mathrm{m}$ diameter).

\section{Guided-wave frequency doubling}

Efficient second harmonic generation (SHG) from 1064 to $532 \mathrm{~nm}$ light have been realized through fs-laser written cladding waveguides in $\mathrm{KTiOPO}_{4}$ and $\mathrm{BiB}_{3} \mathrm{O}_{6}$ crystals based on the phase matching mechanisms. Under pulsed laser pump, the SHG conversion reaches a value as high as $45 \%$ for $\mathrm{KTiOPO}_{4}$ waveguide, and $25 \%$ for the $\mathrm{BiB}_{3} \mathrm{O}_{6}$ waveguide. The nonlinear cladding waveguides have shown superior properties for frequency doubling to the stress induced Type II waveguides.

In summary, our research results have shown that cladding waveguides in dielectric crystals may be successful candidates for diverse applications in photonic systems.

[1] E. J. Murphy, Integrated optical circuits and components: Design and applications (Marcel Dekker, 1999).

[2] S. Juodkazis, V. Mizeikis, and H. Misawa, "Three-dimensional microfabrication of materials by femtosecond lasers for photonics applications," J. Appl. Phys. 106, 051101 (2009).

[3] K. M. Davis, K. Miura, N. Sugimoto, and K. Hirao, "Writing waveguides in glass with a femtosecond laser," Opt. Lett. 21, 1729-1731 (1996).

[4] J. Burghoff, S. Nolte, and A. Tünnermann, "Origins of waveguiding in femtosecond laser structured $\mathrm{LiNbO}_{3}$," Appl. Phys. A 89, 127-132 (2007).

[5] T. Calmano, J. Siebenmorgen, O. Hellmig, K. Petermann, and G. Huber, "Nd:YAG waveguide laser with $1.3 \mathrm{~W}$ output power, fabricated by direct femtosecond laser writing,” Appl. Phys. B 100, 131-135 (2010).

[6] A. G. Okhrimchuk, A. V. Shestakov, I. Khrushchev, and J. Mitchell, "Depressed cladding, buried waveguide laser formed in a YAG:Nd ${ }^{3+}$ crystal by femtosecond laser writing," Opt. Lett. 30, 2248-2250 (2005).

[7] Y. C. Jia, F. Chen, and J. R. Vázquez de Aldana, "Efficient continuous-wave laser operation at $1064 \mathrm{~nm}$ in Nd:YVO $\mathrm{Y}_{4}$ cladding waveguides produced by femtosecond laser inscription,” Opt. Express 20, 16801-16806 (2012).

[8] H. L. Liu, Y. C. Jia, J. R. Vázquez de Aldana, D. Jaque, and F. Chen, "Femtosecond laser inscribed cladding waveguides in Nd:YAG ceramics: Fabrication, fluorescence imaging and laser performance," Opt. Express 20, 18620-18629 (2012).

[9] N. N. Dong, F. Chen, and J .R. Vázquez de Aldana, "Efficient second harmonic generation by birefringent phase matching in femtosecondlaser-inscribed KTP cladding waveguides," Phys. Status Solidi: RRL 6, 306-308 (2012).

[10] Y. C. Jia, J. R. Vázquez de Aldana, C. Romero, Y. Y. Ren, Q. M. Lu, and F. Chen, "Femtosecond-Laser-Inscribed $\mathrm{BiB}_{3} \mathrm{O}_{6} \mathrm{Nonlinear}$ Cladding Waveguide for Second-Harmonic Generation,” Appl. Phys. Express 5, 072701 (2012). 